Chinese Journal of Organic Chemistry

\title{
异伍兹烷衍生物的研究进展
}

\author{
公绪滨 孙成辉* 庞思平* 张 静 李玉川 赵信岐
}

(北京理工大学 材料学院 北京 100081)

\begin{abstract}
摘要 六硝基六氮杂异伍兹烷(HNIW 或 CL-20)是当今世界上综合性能最好的单质炸药，在 20 余年的研究过程中，各 国研究者合成出了百余种异伍兹烷衍生物. 根据笼体上取代基的不同以及合成反应的类型，系统总结了各类衍生物的 合成、用途及反应性. 最后对异伍兹烷衍生物的发展方向进行了展望.
\end{abstract}

关键词 异伍兹烷衍生物; 高能量密度化合物; 综述

\section{Research Progress in Study of Isowurtzitane Derivatives}

\author{
Gong, Xubin \\ Sun, Chenghui* \\ Pang, Siping* \\ Zhang, Jing \\ Li, Yuchuan \\ Zhao, Xinqi \\ (School of Material Science and Engineering, Beijing Institute of Technology, Beijing 100081)
}

\begin{abstract}
Hexanitrohexaazaisowurtzitane (HNIW or CL-20) is presently the most powerful energetic compound in the world. The synthesis of CL-20 or cage molecules has attracted considerable interest of experts in the area of high-energy materials over the last two decades, and more than 100 isowurtzitane derivatives were synthesized. In this paper, the synthesis, application and reactivity of these compounds are summarized systematically according to different substituents on the cage and types of reactions. The developing tends of isowurtzitane derivatives are also presented.

Keywords isowurtzitane derivatives; high energy density compound; review
\end{abstract}

高能量密度化合物(HEDC)一般是指密度达 2.0 $\mathrm{g} \cdot \mathrm{cm}^{-3}$ 、爆速达 $9.5 \mathrm{~km} \cdot \mathrm{s}^{-1}$ 、爆压达 $42 \sim 43 \mathrm{GPa}$ 的含能 化合物 ${ }^{[1]}$. 自美国于 20 世纪 80 年代提出 $\mathrm{HEDC}^{[2]}$ 的概念 以来，各国含能材料工作者在这方面进行了大量的研究 工作, 发现要提高化合物的密度, 含能化合物的设计合 成由平面单环向立体笼形发展是一个趋势. 于是六硝基 六氮杂异伍兹烷(HNIW，俗称 CL-20) ${ }^{[3]}$ 、八硝基立方烷 $(\mathrm{ONC})^{[4]}$ 等化合物相继问世.

CL-20 系统名为 2,4,6,8,10,12-六硝基- 2,4,6,8,10,12六氮杂四环 $\left[5 \cdot 5 \cdot 0.0^{5,9} \cdot 0^{3,11}\right]$ 十二烷, 简称为六硝基六氮 杂异伍兹烷, 为异伍兹烷的衍生物, 于 1987 年由美国海 军武器中心的 Nielsen 首次合成, 成为高能密度化合物 领域的突破, 被誉为含能化合物合成史上的一个里程 碑. $\varepsilon$-CL-20 的晶体密度达 $2.044 \mathrm{~g} \cdot \mathrm{cm}^{-3}$, 标准生成焓约 为 $900 \mathrm{~kJ} \cdot \mathrm{kg}^{-1}, \varepsilon-\mathrm{CL}-20$ 的最大爆速及爆压可分别达 9.5 9.6 km•s${ }^{-1}$ 及 $43 \sim 44 \mathrm{GPa}$, 能量输出可比王牌炸药 奥克托今 $(\mathrm{HMX})$ 高 $10 \% \sim 15 \%{ }^{[5]}$, 是目前综合性能最好
的单质炸药. CL-20 在高能固体推进剂、低特征信号推 进剂、高能混合炸药、发射药等领域有着广阔的应用前 景.

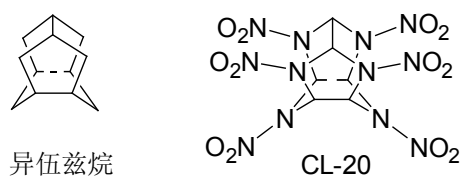

CL-20 以其高密度、高爆速、高爆压、低感度的优 良综合性能，迅速吸引了各国含能材料工作者的兴趣， 对其合成进行了详尽的研究. 此过程中通过缩合、氢解、 氧化和硝解等反应合成了大量新的异伍兹烷衍生物. 同 时, 作为含能化合物, 笼形结构有着独特的优势, 不但 可以提高化合物的密度，而且笼体自身的张力能提高化 合物的能量密度. 因此, 各国研究者对异伍兹烷的含氮 或者含氧衍生物也进行了大量研究，以期获得新的笼形 高能量密度化合物. 在迄今二十余年的研究过程中, 已

\footnotetext{
*E-mail: sunch@bit.edu.cn; pangsp@bit.edu.cn

Received April 22, 2011; revised July 19, 2011; accepted October 25, 2011.

Project supported by the Program for New Century Excellent Talents in University.

教育部新世纪优秀人才支持计划(No. NCET-09-0046)资助项目.
} 
合成出百余种异伍兹烷的衍生物. 这些衍生物中, 有的 可作为制备 CL-20 的中间体; 有的则可能是潜在的高能 量密度化合物; 还有研究发现, 某些衍生物具有生理活 性, 在抑制癌症方面也有着潜在的效果. 而异伍兹烷衍 生物的合成方法则可以为制备其他笼形结构化合物提 供参考.

按照笼体上的取代基不同及制备衍生物的反应类 型, 详细介绍各类衍生物及其特点.

\section{1 烷基类异伍兹烷及其合成}

通过缩合反应构建异伍兹烷笼体结构是合成异伍 兹烷衍生物的必要步骤, 但目前仅烷基取代伯胺可以与 乙二醛发生此反应. 所有烷基类异伍兹烷能量都很低, 不能称之为含能化合物, 它们大多是作为氢解、氧化反 应的前体, 是制备 CL-20 及其他异伍兹烷衍生物的重要 中间体. 目前所有的烷基类异伍兹烷都是通过取代伯胺 与乙二醛的缩合反应制得的, 这类反应的特点是反应条 件温和. 其中所用胺基取代基团主要包括苄基类、其他 芳香基团类以及直链烷基类.

\section{1 苄基类异伍兹烷衍生物}

1989 年, Nielsen 等 $^{[6]}$ 公布了化合物 1 的合成方法, 利用芐胺和乙二醛在温和的条件下, 以 $80 \%$ 的得率得到 1 (Eq. 1). 化合物 1 是 CL-20 制备中的重要中间体, 它可 以进行氢解反应得到 28 进而得到 $\mathbf{3 2}$, 最终硝化得到 CL-20(详见第 2.1 节), 也可以通过氧化反应得到制备 CL-20 的中间体(详见第 2.2 节).

$$
6 \mathrm{C}_{6} \mathrm{H}_{5} \mathrm{CH}_{2} \mathrm{NH}_{2}+3(\mathrm{CHO})_{2} \longrightarrow
$$

此外, Nielsen 等 ${ }^{[3]}$ 以苯环上的氢被取代后的茮胺与 乙二醛反应, 得到了化合物 2 7 (Eq. 2). 但产物得率均 低于化合物 1. 经验证, 化合物 $1 \sim 7$ 在非质子溶剂中均 能稳定存在, 而在甲酸中会很快分解生成油状物.

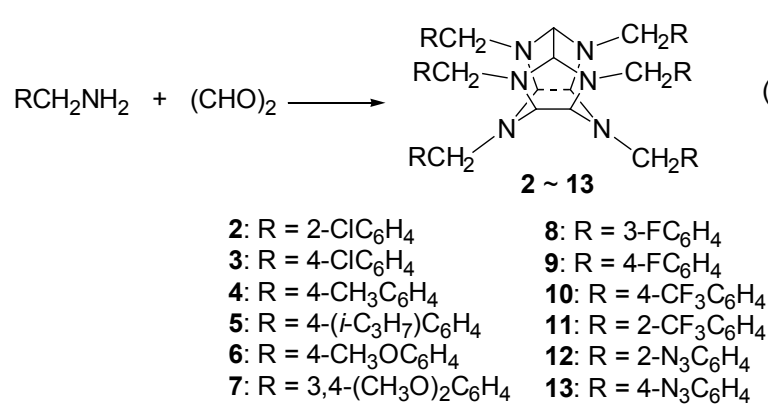

2003 年, Klapötke 等 ${ }^{[7]}$ 用 $\mathrm{F}, \mathrm{CF}_{3}, \mathrm{~N}_{3}$ 等取代苄胺中苯
环上的氢后与乙二醛缩合, 得到了化合物 $\mathbf{8} \sim \mathbf{1 3}$ (Eq. 2). 但得率均较低, 其中晶体得率最高 34\% (8), 最低 $3 \%$ (12). 作者本想比较吸电子基团对此类缩合反应的影响, 但没有得到明确的规律. 此外, 作者在合成化合物 $\mathbf{1 2}$ 时, 意外得到一种副产物 14, 并对其进行了详细的表 征.

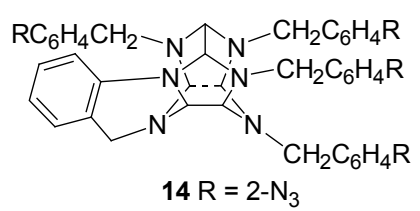

2006 年, Kerscher 与 Klapötke 等 ${ }^{[8]}$ 用多个氟原子取 代芐胺中苯环上的氢原子后, 合成出化合物 $15 \sim 17$, 产 率分别为 $15 \%, 6 \%, 5 \%$, 并且得出结论, 随着氟含量的 增加，化合物的产率越来越低，分离也越来越困难(Eq. 3).

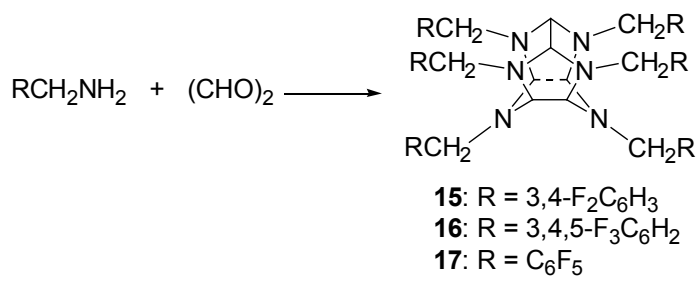

2010 年, Gottlieb 等 ${ }^{[9]}$ 以 $19.7 \%$ 的得率合成出化合物 18,18 能在甲酸乙酯中重排, 得到一种新的笼形化合物 (Scheme 1).

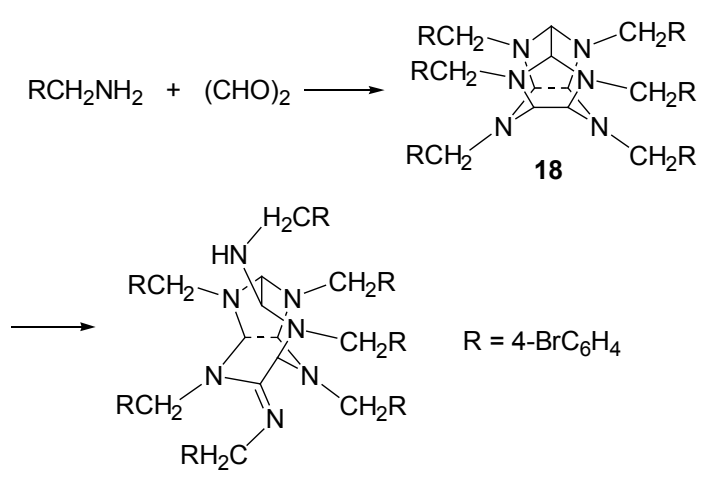

\section{Scheme 1}

由取代芐胺与乙二醛的缩合情况来看, 相对于未取 代芐胺，随着苯环上取代基的增加或者取代基吸电子能 力的增强, 缩合产率呈下降趋势, 同时笼体变的越来越 不稳定.

\section{2 其它芳香基团类异伍兹烷衍生物}

2004 年, Cagnon 等 ${ }^{[10]}$ 利用 2-呋喃甲胺等芳香胺与 乙二醛缩合得到了化合物 19 24, 其产率与化合物 8 13 相比较高, 如 23 的产率可达到 $60 \%$, 但与化合物 1 的产率比还是相差较大(Eq. 4). 另外, 作者还将化合物 
23 在 $100 \%$ 硝酸中进行硝化，以 $12 \%$ 的得率制得了 CL-20. 传统上, 呋喃甲基并不是硝化反应中好的离去 基团, 因此得率应很低, 产品纯度也较差, 但这或许可 以在探索 CL-20 的新合成方法中给我们一些启示.

$$
\mathrm{RNH}_{2}+(\mathrm{CHO})_{2} \longrightarrow
$$

19: $\mathrm{R}=2$-thienyl- $\mathrm{CH}_{2}$

20: $\mathrm{R}=3$-pyridyl- $\mathrm{CH}_{2}$

21: $\mathrm{R}=1$-naphtyl- $\mathrm{CH}_{2}$

22: $\mathrm{R}=\mathrm{CH}_{2} \mathrm{CH}=\mathrm{CHPh}$

23: $\mathrm{R}=2$-furfuryl- $\mathrm{CH}_{2}$

24: $\mathrm{R}=p-\mathrm{ClC}_{6} \mathrm{H}_{4} \mathrm{SO}_{2}$

\section{3 直链烷烃类异伍兹烷衍生物}

2004 年, Cagnon 等 ${ }^{[10]}$ 在甲酸存在条件下, 用烯丙胺 与乙二醛缩合，制得了六烯丙基六氮杂异伍兹烷(25), 得率为 $20 \%$. 作者直接将 25 硝化, 得到少量黄色固体, 经核磁共振和高效液相色谱鉴定为 CL-20. 李新乐 ${ }^{[11]}$ 、 Adamiak 等 ${ }^{[12]}$ 相继研究了 25 的合成, 将其得率提高到 $66.5 \%$.

2008 年, Chapman 等 ${ }^{[13]}$ 在叔丁醇钾的作用下, 将 25 重排, 得到 26, 作者尝试在各种条件下对 26 进行光氧 化，但始终得到成分复杂的混合物. 以 $98 \% \sim 100 \%$ 的发 烟硝酸直接硝化该混合物, 得到少量 CL-20(产率低于 10\%). 将 26 直接进行硝化, 得到 CL-20, 但产率只有 11.6\%. 事实证明, 烯丙基和丙烯基都不是好的硝化离 去基团. 但将烯丙基重排、氧化然后再硝化，则开辟了 一条不用苠胺、不用贵金属催化剂的 CL-20 合成路线. 关于此类反应值得继续探索, 重点应是寻找提高得率的 方法和新反应试剂的开发.

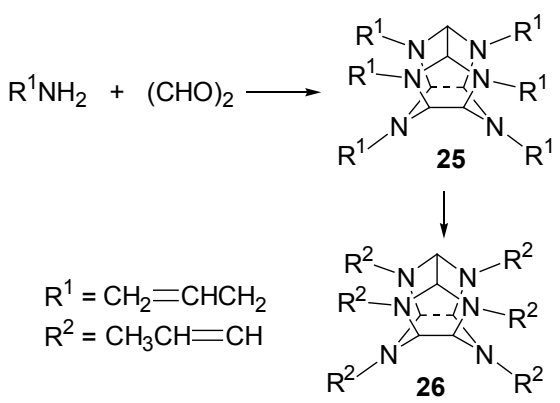

Scheme 2

此外, Chapman 等 ${ }^{[13]}$ 还以炔丙胺与乙二醛反应, 制 备了 27, 产率为 $17 \%(\mathrm{Eq} .5$ ). 虽然 27 无法作为 CL-20 的合成中间体, 但我们认为可以利用端炔的 Click 反应 来制备新型的六氮杂异伍兹烷衍生物.

\section{2 酰基类异伍兹烷及其合成}

烷基类异伍兹烷均不宜作为直接硝化制备 CL-20

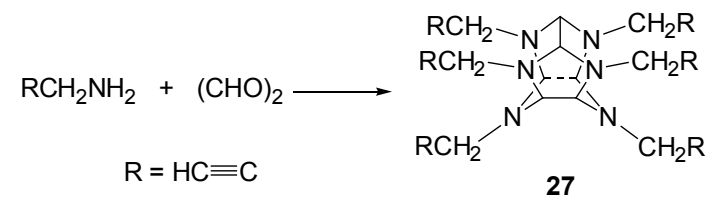

的前体，如 1 在酸性介质中不稳定，极易破环，且苠基 芳环比笼上叔胺具有更强的硝化竞争能力, 因此不能直 接硝解生成 CL-20. 由于酰基的吸电子作用, 酰基类异 伍兹烷衍生物的稳定性大大增强，同时，酰胺中的酰基 是硝化反应中好的离去基团，将烷基转化为易于硝解的 酰基，有助于进一步合成含能异伍兹烷化合物. 其中涉 及的反应类型主要包括氢解反应、氧化反应、酰化反应 等.

\section{1 氢解反应}

制备异伍兹烷的氢解反应可分为一次氢解和二次 氢解. 所谓一次氢解, 就是在酰化剂的存在下, 以钯作 为催化剂, 将 1 笼体上面的四个苠基转化为酰基.

Nielsen 在最初合成 CL-20 过程中, 以乙酸酐为酰化 试剂, $\mathrm{Pd}(\mathrm{OH})_{2} / \mathrm{C}$ 为催化剂, 在溴苯的存在下, 将 $\mathbf{1}$ 进行 一次氢解得到 TADB (28) (Eq. 6). 28 是合成 CL-20 的重 要中间体, 可以作为二次氢解的原料, 根据二次氢解中 所用酰化试剂的不同得到 32, 34 等化合物(Eq. 7); 也可 以作为氧化前体得到四乙酰基二苯甲酰基六氮杂异伍 兹烷等; 还可以直接进行亚硝化、硝化得到 CL-20.

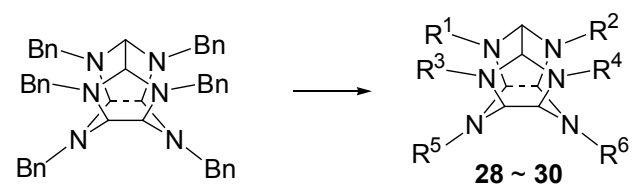

28: $R^{1}=R^{2}=R^{3}=R^{4}=\mathrm{CH}_{3} \mathrm{CO}, R^{5}=R^{6}=\mathrm{C}_{6} \mathrm{H}_{5} \mathrm{CH}_{2}$ 29: $R^{1}=R^{2}=R^{3}=\mathrm{CH}_{3} \mathrm{CO}, \mathrm{R}^{4}=\mathrm{R}^{5}=\mathrm{R}^{6}=\mathrm{C}_{6} \mathrm{H}_{5} \mathrm{CH}_{2}$ 30: $R^{1}=R^{4}=\mathrm{CH}_{3} \mathrm{CO}, \mathrm{R}^{2}=\mathrm{R}^{3}=\mathrm{R}^{5}=\mathrm{R}^{6}=\mathrm{C}_{6} \mathrm{H}_{5} \mathrm{CH}_{2}$

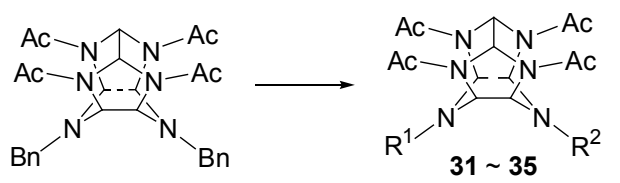

31: $R^{1}=R^{2}=\mathrm{CH}_{3} \mathrm{CO} ; 32: \mathrm{R}^{1}=\mathrm{R}^{2}=\mathrm{H} ; 33: \mathrm{R}^{1}=\mathrm{R}^{2}=\mathrm{CH}_{3} \mathrm{CH}_{2}$; 34: $\mathrm{R}^{1}=\mathrm{R}^{2}=\mathrm{CHO} ; 35: \mathrm{R}^{1}=\mathrm{C}_{6} \mathrm{H}_{5} \mathrm{CH}_{2}, \mathrm{R}^{2}=\mathrm{H}$

2004 年，韩卫荣等 ${ }^{[14]}$ 通过控制 $\mathbf{1}$ 的氢解程度，得到 了氢解反应的中间体 29, 并对其晶体结构进行了表征. 2005 年，黄兴等 ${ }^{[15]}$ 用同样的方法得到了化合物 30. 这 对进一步了解和证实氢解脱苠反应机理起到了重要作 
用. 作者还对 28 和 29 的氢解特性进行了比较 ${ }^{[16]}$, 得出 结论认为: 29 稳定性差, 在甲酸介质中易于破笼, 且易引 起二次氢解催化剂中毒.

在 Nielsen 首次合成 $\mathbf{2 8}$ 时就发现, 延长反应时间并 不能把笼体下面的两个苠基脱掉, 要想将下面的两个苠 基脱掉，只能改变反应体系，进行二次氢解.

Wardle 等 ${ }^{[17]}$ 先将 28 进行氢解, 氢解产物再与氢氧 化钠、吡啶反应, 得到化合物 31, 31 可以作为 CL-20 的 硝化前体, 在各种超酸体系中合成 CL-20 $0^{[18]}$. 欧育湘等 [19] 将 28 用 $\mathrm{Pd}(\mathrm{OH})_{2} / \mathrm{C}$ 催化剂(含钯 $20 \%$ ) 在乙酸中氢解, 反应完成后, 过滤分离出催化剂, 将乙酸溶液中加入乙 酰氯, 再升温至 $48 \sim 55{ }^{\circ} \mathrm{C}$, 反应 $20 \sim 30 \mathrm{~min}$, 减压蒸除 溶剂, 往剩余物中加入无水乙醇, 过滤, 也可得 31 .

1995 年, 赵信岐教授 ${ }^{[20]}$ 在乙酸介质中, 用钯负载催 化剂对 28 进行二次氢解, 制备了 TAIW (32). 日本也在 1996 年合成出了此前体 ${ }^{[21]} .32$ 可以直接作为 CL-20 的硝 化前体, 在当今工业生产中应用最为广泛.

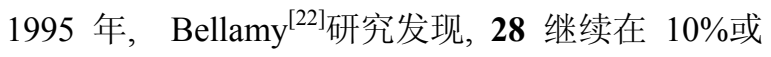
$20 \%$ 的乙酸/乙酸䣶介质中氢解, 可以得到化合物 33 . 1999 年欧育湘等 ${ }^{[23]}$ 在 $V$ (乙酸䣶) $/ V($ 水 $)=80 / 15$ 的溶剂 中, 将 28 用 $\mathrm{Pd}(\mathrm{OH})_{2} / \mathrm{C}$ 催化氢解也可制得 33 , 产率 $85 \%$ (Eq. 7).

1997 年, Wardle 等 ${ }^{[24]}$ 以甲酸为介质, 氢解 28 得到 TADF (34), 产率 $86 \%$ (Eq. 7). 34 也是制备 CL-20 的硝化 前体, 2002 年前曾应用于美国的工业化生产中.

1999 年, 欧育湘等 ${ }^{[23]}$ 以 28 为原料, 分别在甲酸和 乙酸中氢解, 得到化合物 34 和 35 , 产率分别为 $79 \%$ 和 90\% (Eq. 7).

2005 年, 黄兴 ${ }^{[15]}$ 分别以 $\mathbf{2 9 , 3 0}$ 为原料, 在甲酸的存 在下, 进行二次氢解制得了化合物 $\mathbf{3 6}$ 和 $\mathbf{3 7}$, 并研究了 不同实验因素对产率的影响. 36 和 37 均可作为 CL-20 的硝化前体直接得到 CL-20, 但得率和纯度都较差.

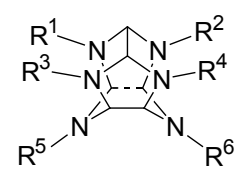

36: $\mathrm{R}^{1}=\mathrm{R}^{2}=\mathrm{R}^{3}=\mathrm{CH}_{3} \mathrm{CO}, \mathrm{R}^{4}=\mathrm{R}^{5}=\mathrm{R}^{6}=\mathrm{CHO}$ 37: $R^{1}=R^{4}=\mathrm{CH}_{3} \mathrm{CO}, \mathrm{R}^{2}=\mathrm{R}^{3}=\mathrm{R}^{5}=\mathrm{R}^{6}=\mathrm{CHO}$

\section{2 氧化反应}

由于氢解反应需要消耗大量昂贵的钯催化剂, 大幅 度增加了 CL-20 的合成成本, 因此, 有研究者尝试使用 廉价的高镇酸钾、臭氧等氧化剂, 将芐基氧化脱芐或者 转化成易于硝解的官能团, 主要氧化底物为 $\mathbf{1}$ 和 28.

\subsection{1 以 HBIW 为底物的氧化反应}

1999 年, 邱文革等 ${ }^{[25}$ 将 1 在三氧化铬/乙酸䣶体系
中氧化得到化合物 38, 但得率仅有 $18 \%$ (Eq. 8). 研究可 知 38 是一种非常稳定的化合物, 但遗憾的是苯甲酰基 很难硝解脱掉 ${ }^{[26]}$, 因此不能作为 CL-20 的合成前体.

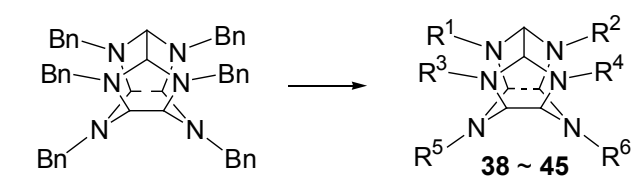

38: $R^{1}=R^{2}=R^{3}=R^{4}=R^{5}=R^{6}=C_{6} H_{5} C O$

39: $R^{1}=\mathrm{CH}_{3} \mathrm{CO}, \mathrm{R}^{2}=\mathrm{R}^{3}=\mathrm{R}^{4}=\mathrm{C}_{6} \mathrm{H}_{5} \mathrm{CO}, \mathrm{R}^{5}=\mathrm{R}^{6}=\mathrm{C}_{6} \mathrm{H}_{5} \mathrm{CH}_{2}$

40: $R^{1}=R^{4}=\mathrm{C}_{6} \mathrm{H}_{5} \mathrm{CO}, \mathrm{R}^{2}=\mathrm{R}^{3}=\mathrm{CH}_{3} \mathrm{CO}, \mathrm{R}^{5}=\mathrm{R}^{6}=\mathrm{C}_{6} \mathrm{H}_{5} \mathrm{CH}_{2}$

41: $R^{1}=R^{4}=R^{5}=R^{6}=\mathrm{C}_{6} \mathrm{H}_{5} \mathrm{CH}_{2}, R^{2}=R^{3}=\mathrm{C}_{6} \mathrm{H}_{5} \mathrm{CO}$

42: $R^{1}=R^{5}=R^{6}=\mathrm{C}_{6} \mathrm{H}_{5} \mathrm{CH}_{2}, \mathrm{R}^{2}=\mathrm{R}^{3}=\mathrm{R}^{4}=\mathrm{C}_{6} \mathrm{H}_{5} \mathrm{CO}$

43: $\mathrm{R}^{5}=\mathrm{R}^{6}=\mathrm{C}_{6} \mathrm{H}_{5} \mathrm{CH}_{2}, \mathrm{R}^{1}=\mathrm{R}^{2}=\mathrm{R}^{3}=\mathrm{R}^{4}=\mathrm{C}_{6} \mathrm{H}_{5} \mathrm{CO}$

44: $\mathrm{R}^{6}=\mathrm{C}_{6} \mathrm{H}_{5} \mathrm{CH}_{2}, \mathrm{R}^{1}=\mathrm{R}^{2}=\mathrm{R}^{3}=\mathrm{R}^{4}=\mathrm{R}^{5}=\mathrm{C}_{6} \mathrm{H}_{5} \mathrm{CO}$

45: $\mathrm{R}^{1}=\mathrm{R}^{2}=\mathrm{R}^{3}=\mathrm{CH}_{3} \mathrm{CO}, \mathrm{R}^{4}=\mathrm{C}_{6} \mathrm{H}_{5} \mathrm{CO}, \mathrm{R}^{5}=\mathrm{R}^{6}=\mathrm{C}_{6} \mathrm{H}_{5} \mathrm{CH}_{2}$

2000 年, 陈树森等 ${ }^{[27]}$ 采用高锰酸钾/乙酸䣶体系对 1 进行氧化, 得到化合物 39, 40, 得率分别为 $14 \%$ 和 $10 \%$, 并对其结构进行了表征, 并且作者发现加入碳酸 钠能提高 39, 40 的产率(Eq. 8).

2002 年, 邱文革等 ${ }^{[28]}$ 改用高锰酸钾/芐基三甲基碘 化铵/二氯甲烷体系, 对 $\mathbf{1}$ 进行氧化, 得到了化合物 $\mathbf{4 1}$ 44 的混合物, 总得率约为 $85 \%$, 并分别对其进行了分离 表征(Eq. 8), 另外对化合物 $\mathbf{4 1}$ 的晶体结构进行了详细归 属 ${ }^{[29]}$. 化合物 $\mathbf{4 1} \sim \mathbf{4 4}$ 均可以在三氧化铬/乙酸䣶体系中 高得率得到化合物 $\mathbf{3 8}$.

2002 年, 庞思平等 ${ }^{[30]}$ 采用乙酸䣲/DMF 为介质, 在 四乙基溴化铵的催化下用高锰酸钾氧化 $\mathbf{1}$, 得到化合物 39, 40, 45 (Eq. 8), 并对其反应机理进行了讨论. 相对于 单纯的高锰酸钾/乙酸䣶体系, 相转移催化剂的加入改 善了高锰酸钾的氧化效果.

2003 年, 刘江强等 ${ }^{[31]}$ 以过硫酸铵作为氧化剂, 乙酸 䣶作为反应介质, 在硝酸铈铵的催化下成功将 $\mathbf{1}$ 氧化为 28, 但得率仅有 $8.1 \%$.

2005 年, 庞思平等 ${ }^{[32]}$ 改用乙酸䣲为介质, 在四乙基 澳化铵和 NBS 存在条件下, 用高镇酸钾对 $\mathbf{1}$ 进行氧化, 得到了化合物 $28,39,40,45$ 的混合物. 这 4 种化合物都 可以进行亚硝化、硝化制备 CL-20.

2010 年, 李亚裕等 ${ }^{[33]}$ 以高锰酸钾/二氯甲烷/碳酸钠 体系氧化 1 , 得到化合物 $\mathbf{4 3}$, 而改变氧化体系为高镇酸 钾/乙酸酐/碳酸钠时, 得到化合物 $\mathbf{2 8}, \mathbf{3 9}, \mathbf{4 0}, \mathbf{4 5}$ (Eq. 8).

由此可知, 1 在不同的氧化体系下, 随着氧化程度 和酰化试剂的不同, 可以得到不同的产物. 比较三种不 同的氧化剂, 三氧化铬氧化能力较弱; 高锰酸钾氧化能 力略强, 但仍需在催化剂作用下进行反应, 且氧化并不 彻底; 过硫酸铵氧化最为彻底, 但产率很低, 而且催化 剂硝酸铈铵价格昂贵. 


\subsection{2 以 $\mathrm{TADB}$ 为底物的氧化反应}

2002 年, 刘江强等 ${ }^{[34]}$ 采用高锰酸钾作为氧化剂, 乙 酸䣶作为反应介质, 在三氟化硼的催化下对 28 进行氧 化脱茮, 得到化合物 31, 46, 47 (Eq. 9). 这是第一次通过 氧化法得到 31. 46, 47 都可以作为亚硝化前体.

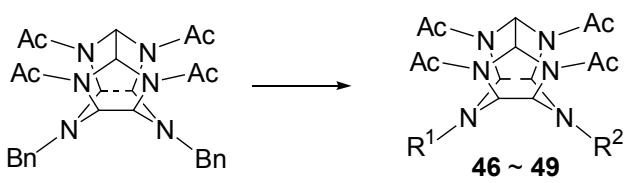

46: $\mathrm{R}^{1}=\mathrm{R}^{2}=\mathrm{C}_{6} \mathrm{H}_{5} \mathrm{CO} ; 47: \mathrm{R}^{1}=\mathrm{C}_{6} \mathrm{H}_{5} \mathrm{CO}, \mathrm{R}^{2}=\mathrm{C}_{6} \mathrm{H}_{5} \mathrm{CH}_{2}$;

48: $R^{1}=R^{2}=N_{2} ; 49: R^{1}=N_{2}, R^{2}=\mathrm{C}_{6} \mathrm{H}_{5} \mathrm{CH}_{2}$

2003 年, 刘江强等 ${ }^{[31]}$ 以过硫酸铵作为氧化剂, 乙酸 酐作为反应介质, 在硝酸铈铵催化下对 $\mathbf{2 8}$ 进行氧化, 得 到化合物 48 和 49, 产率分别为 66.4\%和 $11.7 \%$ (Eq. 9). 这两个化合物都可以直接硝化得到 CL-20.

无论是以 1 还是 28 为氧化底物, 得到的部分氧化产 物都可以作为亚硝化或者硝化前体直接制备 CL-20, 从 而避免了使用贵金属催化剂. 但缺点是氧化产物成分较 复杂, 得率也不高. 因此, 此类反应的重点应是提高氧 化体系的选择性及产率.

\section{3 酰化反应}

利用酰化反应直接得到新的异伍兹烷衍生物基本 是以 32 笼底的两个仲胺基为修饰点.

2000 年, 刘利华等 ${ }^{[35]}$ 以 32 为底物, 以 DMF 为溶剂, 与氯乙酰氯反应，制得了化合物 $\mathbf{5 0}$, 产率为 $89.7 \%, \mathbf{5 0}$ 在硝硫混酸中以 $94.1 \%$ 的得率硝化得到化合物 $\mathbf{5 7}$, 并分 离出其副产物 58 (Scheme 3).

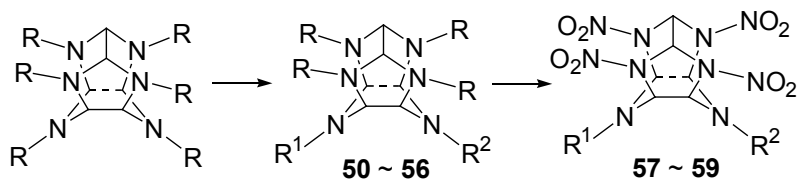

50: $\mathrm{R}=\mathrm{CH}_{3} \mathrm{CO}, \mathrm{R}^{1}=\mathrm{R}^{2}=\mathrm{CH}_{2} \mathrm{ClCO}$

51: $\mathrm{R}=\mathrm{CH}_{3} \mathrm{CO}, \mathrm{R}^{1}=\mathrm{R}^{2}=\mathrm{CH}_{3} \mathrm{CH}_{2} \mathrm{CO}$

52: $\mathrm{R}=\mathrm{CH}_{3} \mathrm{CO}, \mathrm{R}^{1}=\mathrm{R}^{2}=\mathrm{CH}_{3} \mathrm{CH}_{2} \mathrm{CH}_{2} \mathrm{CO}$

53: $\mathrm{R}=\mathrm{CH}_{3} \mathrm{CO}, \mathrm{R}^{1}=\mathrm{R}^{2}=i-\mathrm{PrCO}$

54: $\mathrm{R}=\mathrm{CH}_{3} \mathrm{CH}_{2} \mathrm{CO}, \mathrm{R}^{1}=\mathrm{R}^{2}=\mathrm{CH}_{3} \mathrm{CH}_{2} \mathrm{CH}_{2} \mathrm{CO}$

55: $\mathrm{R}=\mathrm{CH}_{3} \mathrm{CH}_{2} \mathrm{CO}, \mathrm{R}^{1}=\mathrm{R}^{2}=i-\mathrm{PrCO}$

56: $\mathrm{R}=\mathrm{CH}_{3} \mathrm{CO}, \mathrm{R}^{1}=\mathrm{R}^{2}=4-\mathrm{ClC}_{6} \mathrm{H}_{4} \mathrm{CO}$

57: $\mathrm{R}^{1}=\mathrm{R}^{2}=\mathrm{CH}_{2} \mathrm{ClCO}$

58: $\mathrm{R}^{1}=\mathrm{NO}_{2}, \mathrm{R}^{2}=\mathrm{CH}_{2} \mathrm{ClCO}$

59: $\mathrm{R}^{1}=\mathrm{R}^{2}=3,5-\left(\mathrm{NO}_{2}\right)_{2}-4-\mathrm{ClC}_{6} \mathrm{H}_{2} \mathrm{CO}$

\section{Scheme 3}

2006 年, 刘进全等 ${ }^{[36]}$ 以 32 为原料, 分别在丙酸/丙 酰氯、丁酸/丁酰氯、异丁酸/异丁酰氯体系中缓慢升温 回流，制得了化合物 51 53. 而将反应底物换为四丙酰 基六氮杂异伍兹烷后在丁酸/丁酰氯、异丁酸/异丁酰氯
体系中制备了化合物 54, 55 (Scheme 3). 以上这些衍生 物都可作为硝化前体制备出 CL-20, 但从原子经济性角 度看，都不划算.

2006 年, 吴相国等 ${ }^{[37]}$ 以 32 为底物, 以 DMF 为溶剂, 在无水氯化铝的存在下与对氯苯甲酰氯反应，以 $86.4 \%$ 的得率制得了化合物 56. 化合物 56 在硝硫混酸中硝化 得到化合物 59, 得率为 $71.1 \%$ (Scheme 3 ).

\section{4 质子化反应}

2006 年, Wardle 等 ${ }^{[17]}$ 将 28 分别在甲酸/甲醇、甲酸/ 水、甲酸体系中进行氢解, 分别得到 32, 60, 61 (Scheme 4). 化合物 32 在与氢氧化钠反应后, 在吡啶中与乙酸酐 反应可以得到化合物 31 , 而化合物 60 和 61 分别在吡啶 中与乙酸酐反应得到化合物 $2,4,6,8,12$-五乙酰基-10-甲 酰基-2,4,6,8,10,12-六氮杂异伍兹烷(62)和 34. 化合物 $32,60,61$ 均可作为合成 CL-20 的重要中间体.

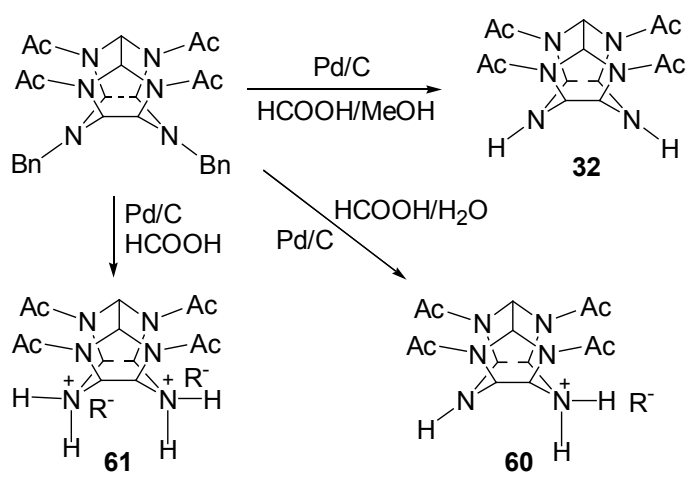

Scheme 4

\section{3 带含能基团异伍兹烷及其合成}

异伍兹烷类化合物主要用途是用作含能化合物，其 中的含能基团是其能量的主要来源，当前应用在异伍兹 烷笼体上的基团主要包括硝基、亚硝基、叠氮基、三硝 基甲基等. 涉及的反应类型有亚硝化反应、硝化反应、 氧化反应等.

\section{1 亚硝化反应}

亚硝化反应大多采用亚硝酸钠/硝酸体系为亚硝化 剂, 多数研究者希望通过一步亚硝化代替二次氢解，从 而降低 CL-20 成本.

Nielsen 在首次合成 CL-20 时就是先将 28 亚硝化脱 茮得到 63, 作为硝化前体. 但当时 Nielsen 所用的试剂 $\mathrm{NOBF}_{4}, \mathrm{NO}_{2} \mathrm{BF}_{4}$ 价格昂贵, 1997 年, Wardle 等 ${ }^{[24]}$ 改用 $\mathrm{N}_{2} \mathrm{O}_{4}$ 作为亚硝化剂，降低了其成本，2003 年，吕连营 等 ${ }^{[38]}$ 用多种方法对 28 进行了亚硝化, 所用试剂均为廉 价的无机试剂, 最高得率为 $82.2 \%$.

除了以 28 为底物外, Kodama 等 ${ }^{[39]}$ 以 32 为底物, 用 亚硝酸钠作亚硝化剂也制得了化合物 63，产率为 95\%, 
并且以 $100 \%$ 硝酸为氧化剂对 63 进行硝化可得到 48. 钱 华等 ${ }^{[40]}$ 则用臭氧作氧化剂实现上述过程.

$$
{ }_{63} \sim 70
$$

63: $\mathrm{R}^{1}=\mathrm{R}^{2}=\mathrm{R}^{3}=\mathrm{R}^{4}=\mathrm{CH}_{3} \mathrm{CO}, \mathrm{R}^{5}=\mathrm{R}^{6}=\mathrm{NO}$

64: $\mathrm{R}^{1}=\mathrm{CH}_{3} \mathrm{CO}, \mathrm{R}^{2}=\mathrm{R}^{3}=\mathrm{R}^{4}=\mathrm{C}_{6} \mathrm{H}_{5} \mathrm{CO}, \mathrm{R}^{5}=\mathrm{R}^{6}=\mathrm{NO}$

65: $\mathrm{R}^{2}=\mathrm{R}^{3}=\mathrm{CH}_{3} \mathrm{CO}, \mathrm{R}^{1}=\mathrm{R}^{4}=\mathrm{C}_{6} \mathrm{H}_{5} \mathrm{CO}, \mathrm{R}^{5}=\mathrm{R}^{6}=\mathrm{NO}$

66: $R^{1}=R^{2}=R^{3}=\mathrm{CH}_{3} \mathrm{CO}, \mathrm{R}^{4}=\mathrm{C}_{6} \mathrm{H}_{5} \mathrm{CO}, \mathrm{R}^{5}=\mathrm{R}^{6}=\mathrm{NO}$

67: $\mathrm{R}^{1}=\mathrm{CH}_{3} \mathrm{CO}, \mathrm{R}^{2}=\mathrm{R}^{3}=\mathrm{R}^{4}=\mathrm{R}^{6}=\mathrm{C}_{6} \mathrm{H}_{5} \mathrm{CO}, \mathrm{R}^{5}=\mathrm{NO}$

68: $R^{1}=\mathrm{CH}_{3} \mathrm{CO}, \mathrm{R}^{2}=\mathrm{R}^{3}=\mathrm{R}^{4}=\mathrm{R}^{5}=\mathrm{R}^{6}=\mathrm{C}_{6} \mathrm{H}_{5} \mathrm{CO}$

69: $\mathrm{R}^{1}=\mathrm{R}^{2}=\mathrm{R}^{3}=\mathrm{R}^{4}=\mathrm{C}_{6} \mathrm{H}_{5} \mathrm{CO}, \mathrm{R}^{5}=\mathrm{R}^{6}=\mathrm{NO}$

70: $R^{1}=R^{2}=R^{3}=R^{4}=R^{5}=C_{6} H_{5} C O, R^{6}=N O$

2002 年, 庞思平等 ${ }^{[41]}$ 以 39, 40, 45 为原料, 采用硝 酸(65\%)/亚硝酸钠体系对其进行亚硝化, 得到化合物 64 66, 产率均大于 $80 \%$. 作者还研究了 $64 \sim 66$ 的硝化 反应性, 发现他们不能耐受强的硝化条件, 但可以在 $\mathrm{HNO}_{3} / \mathrm{NH}_{4} \mathrm{NO}_{3}$ 体系下硝化得到 CL-20, 不过产率均低 于 $25 \%{ }^{[32]}$. 另外, 以 39 为原料时, 还分离出两种副产物 67, 68, 并分别进行了表征 ${ }^{[42]}$.

2003 年, 刘江强等 ${ }^{[43]}$ 以 43 及 44 为底物, 采用硝酸 $(65 \%)$ 亚硝酸钠体系制备了化合物 69, 70, 两者都有望 作为 CL-20 硝化前体.

亚硝基虽然也是含能基团，但在异伍兹烷化合物中 数目很少, 一般和酰基共同存在, 所以其含能性能较差, 主要还是用作制备 CL-20 的中间体.

\section{2 硝化反应}

硝化反应是制备 CL-20 的最后一步, 通常采用硝 酸、硝硫混酸、硝酸-乙酸酤等硝化剂对氢解、氧化以 及亚硝化产物进行硝化, 最终得到 CL-20.

虽然前述的很多化合物都可以通过不同的硝化体 系得到 CL-20, 但大部分都因为产率太低或纯度太低而 不能进行实际应用. 目前能够应用于工业生产的只有 32 和 34. 但是以 34 为前体时, 产品中总会含有副产物 72, 且很难通过重结晶的方法除去, 因此在纯度上不能 符合要求. 而以 32 为前体则不会存在该问题, 因此 32 是目前工业生产中的主要前体.

在 2000, 2001 年的 ICT (The Fraunhofer Institute for Chemical Technology) 年会上美国 Thiokol 公司的 Hamilton $^{[44]}$ 及 Cannizzo ${ }^{[45]}$ 详细研究了 32 的硝化. 发现 32 可以快速平稳的硝化为 CL-20, 得率高(大于 $93 \%$ ), 产品纯度高(大于 99.3\%). 但对于其硝化机理 Hamilton 等 ${ }^{[44]}$ 猜测主要途径是笼底仲胺基氮首先发生了质子化, 阻碍了硝化作用, 于是 $\mathrm{NO}_{2}^{+}$则首先进攻酰基氮, 随着 硝解作用的进行, 反应体系中逐渐增多的乙酸和硝酸形
成乙酰硝酸酯，这种硝化剂再对质子化的仲胺进行硝 化. 简言之，硝化反应途径是先发生酰胺基硝解，后发 生仲胺基硝化的反应历程. Bellamy 等 ${ }^{[46]}$ 则从 CL-20 产 品中恒含 $N$-乙酰基衍生物中间体这一事实出发, 认为 游离胺基比乙酰基应该更容易硝化，但都缺乏直接试验 证明.

方涛等 ${ }^{[47]}$ 发现 32 笼底的两个仲胺基很容易被硝化, 可高收率得到 48. 2009 年，孙成辉等 ${ }^{[48]}$ 在此基础上采用 分阶段终止反应进程的方法，依次分离并表征了 32 硝 化反应中的 5 种中间体 48, 71 74 (Scheme 5), 试验结 果证明 32 在混酸中遵循仲胺基先硝化、乙酰基后硝解, 分步硝化的反应机理.
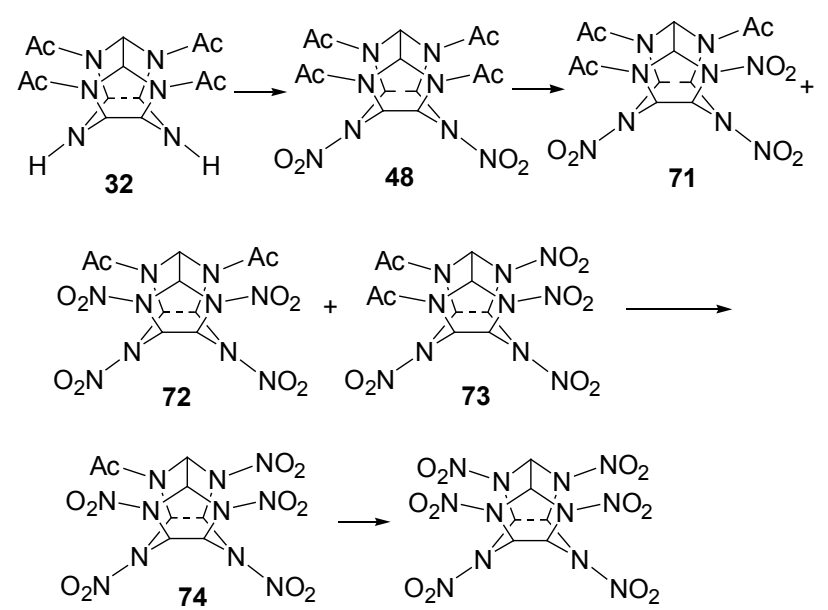

Scheme 5

而对于 34 的硝化，研究发现在不同的硝化体系下， 硝解顺序是不同的. 在硝酸介质中反应时，首先是甲酰 基硝解，然后再是乙酰基. 2008 年，陈留群等 ${ }^{[49]}$ 以无机 盐作催化剂对 34 进行硝化, 并分离出 34 合成 CL-20 的 主要杂质 74, 并得出了合成 74 的最佳条件, 为减少 CL-20 中的杂质提供了依据. 2009 年，贺晶 ${ }^{[50]}$ 通过硝化 34 得到化合物 75 (Scheme 6).

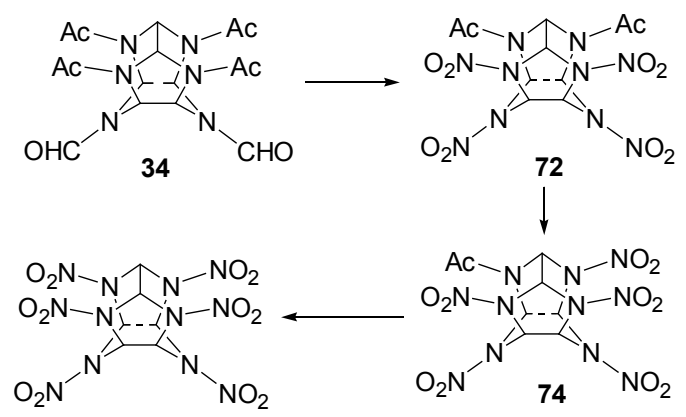

\section{Scheme 6}

而在硝硫混酸介质中时, 硝化顺序则正好相反, 首 先是乙酰基的硝解, 然后才是甲酰基. 2008 年, 施瑞 ${ }^{[51]}$ 
在硝硫混酸中以较高产率制备了 75 , 蔡水兰 ${ }^{[52]}$ 通过控 制硝化程度，在硝硫混酸体系下得到了中间体 76 (Scheme 7).

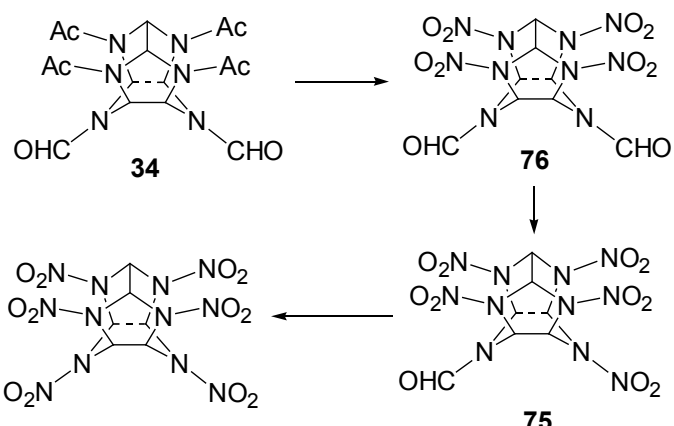

Scheme 7

2000 年，陈树森等 ${ }^{[26]}$ 以 $\mathrm{NH}_{4} \mathrm{NO}_{3} / 65 \% \mathrm{HNO}_{3}$ 为硝解 剂, 对化合物 38 进行硝化, 得到化合物 77, 产率大于 $60 \%$.

$$
\begin{aligned}
& \text { 77: } \mathrm{R}^{1}=\mathrm{R}^{2}=\mathrm{R}^{3}=\mathrm{R}^{4}=\mathrm{NO}_{2}, \mathrm{R}^{5}=\mathrm{R}^{6}=\mathrm{C}_{6} \mathrm{H}_{5} \mathrm{CO} \\
& \text { 78: } \mathrm{R}^{1}=\mathrm{R}^{2}=\mathrm{R}^{3}=\mathrm{R}^{5}=\mathrm{R}^{6}=\mathrm{NO}_{2}, \mathrm{R}^{4}=\mathrm{C}_{6} \mathrm{H}_{5} \mathrm{CO}
\end{aligned}
$$

2005 年, 庞思平等 ${ }^{[32]}$ 用 $\mathrm{HNO}_{3} / \mathrm{NH}_{4} \mathrm{NO}_{3}$ 体系硝化 66 得到化合物 78 .

2010 年, 孙成辉等 ${ }^{[53]}$ 以化合物 79(其合成过程见 Scheme 8) 在 $98 \% \mathrm{HNO}_{3} / \mathrm{Ac}_{2} \mathrm{O}$ 中 $50{ }^{\circ} \mathrm{C}$ 反应 $2 \mathrm{~h}$, 得到化 合物 80,80 继续在硝硫混酸体系中 $80{ }^{\circ} \mathrm{C}$ 反应 $2 \mathrm{~h}$ 得到 化合物 81 (Scheme 8).

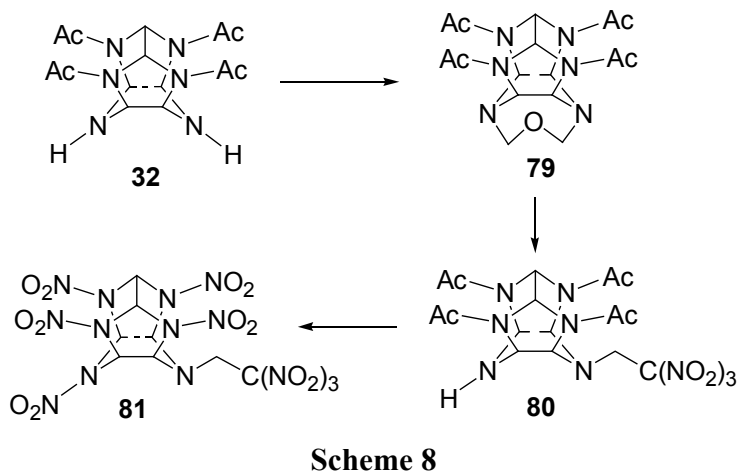

化合物 81 是一种新型的既含有硝基又含有硝仿基 的异伍兹烷衍生物, 其分解温度为 $237{ }^{\circ} \mathrm{C}$ (DSC), 是一 种有应用潜力的含能化合物.

\section{3 选择性硝化}

为了得到不同于 CL-20 的异伍兹烷含能化合物, 需 要采取选择性硝化, 即先将不需要硝化的基团保护起 来, 硝化完成后再进行脱保护.
2007 年, Bellamy 等 ${ }^{[46,54]}$ 希望合成出化合物 82 和 83, 利用笼底六元环上的氨基进行反应.

化合物 82 的合成路线为：用 32 与三氟乙酸酐反应， 得到化合物 84,84 在硝硫混酸中反应得到化合物 85 , 而 后 85 在乙醇/乙酸钠中脱保护得到 82 (Scheme 9).

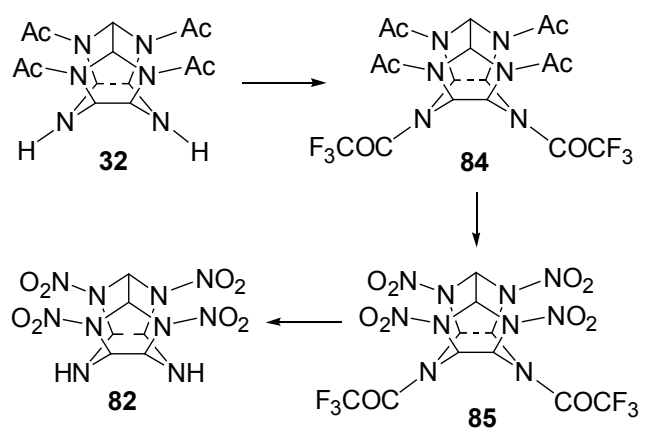

Scheme 9

化合物 83 有两条合成路线: 一条是化合物 84 在乙 醇中脱保护得到化合物 86.86 再进行硝化，得到化合物 87 , 再进行二次脱保护最终得到 83 ; 另一条路线为 32 在乙酸/乙酸酐体系中进行酰化反应得到化合物 $\mathbf{8 8}, \mathbf{8 8}$ 在三氟乙酸酐中回流反应得到化合物 89,89 在硝硫混酸 中硝化得到化合物 87, 进而脱保护得到 83 (Scheme 10).

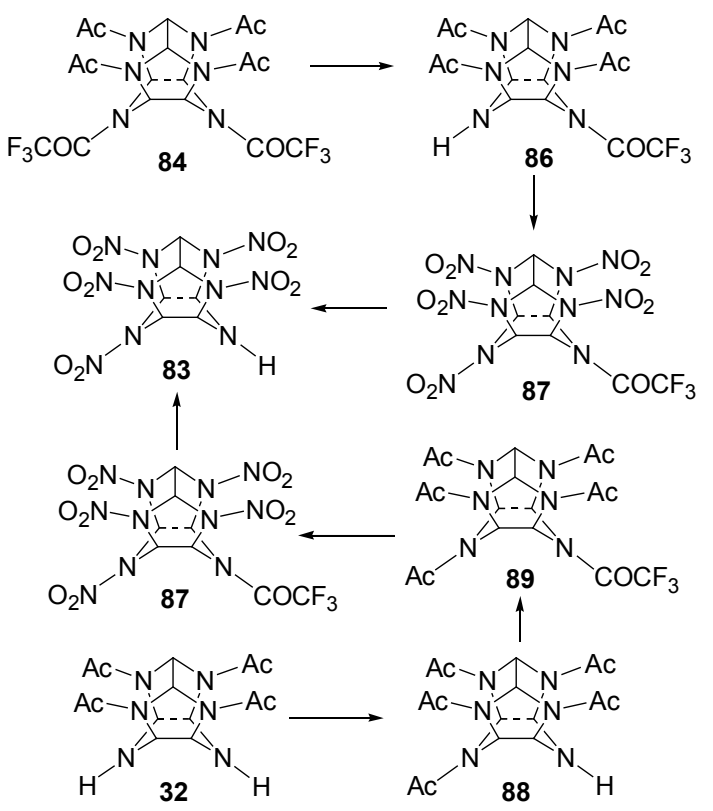

Scheme 10

得到化合物 82 和 83 后，作者利用笼底氨基的活性 进行了一系列反应. 用 $\mathrm{N}_{2} \mathrm{O}_{4}$ 对 82 进行亚硝化, 可以得 到化合物 90.82 分别与 $\mathrm{ClCONCO}, \mathrm{Cl}_{3} \mathrm{CCONCO}$ 反应, 制得了化合物 91, 92. 化合物 92 在硫酸存在下，在甲醇 中回流 $7.5 \mathrm{~h}$ 制得了化合物 93.

化合物 83 分别与 $\mathrm{EtNCO}, \mathrm{ClCONCO} / \mathrm{EtOH}, \mathrm{Cl}_{3} \mathrm{C}$ CONCO 反应，制得了化合物 94 96. 同 92 一样，化合 
物 96 在硫酸存在下, 在甲醇中回流 $2 \mathrm{~h}$ 可以制得化合物 97.

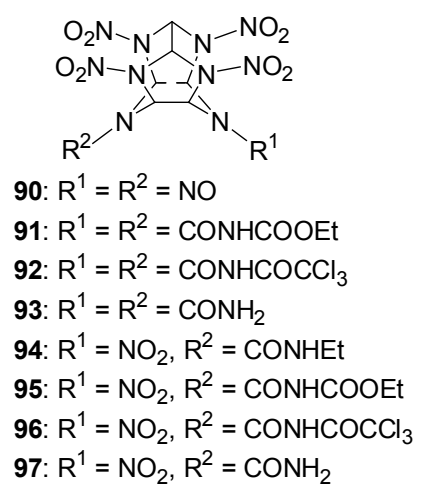

\section{4 还原反应}

2004 年, Lukyanov 等 ${ }^{[55]}$ 用 $\mathrm{SnCl}_{2} \bullet \mathrm{H}_{2} \mathrm{O}$ 还原 CL-20 得到五硝基六氮杂异伍兹烷的两种同分异构体 98 和 99. 98, 99 可以与亚硝化剂 $\mathrm{NOBF}_{4}$ 反应生成化合物 $\mathbf{1 0 0}, \mathbf{1 0 1}$, 而 100, 101 与盐酸反应又可生成 98,99 (Scheme 11).

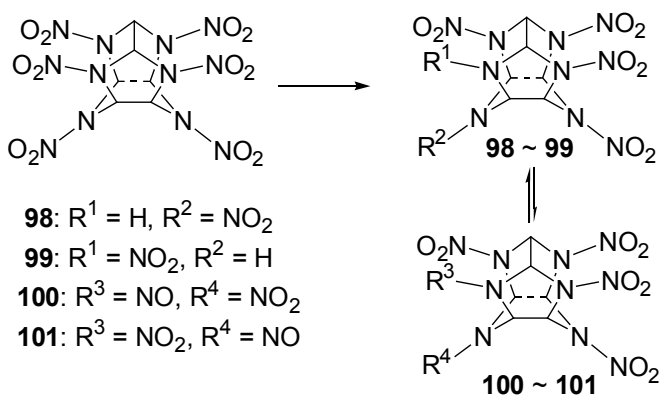

\section{Scheme 11}

2005 年, Duddu 等 ${ }^{[56]}$ 以乙酸乙酯为溶剂, 在 $\mathrm{Pd} / \mathrm{C}$ 催 化剂作用下用氢气还原 CL-20, 得到化合物 98, 产率为 $58 \%$.

\section{5 亲核取代反应}

2004 年, 吴玉凯等 ${ }^{[57]}$ 将 $\mathbf{5 7}$ 在室温下与 $\mathrm{NaN}_{3}$ 反应得 到产物 102 (Eq. 10).

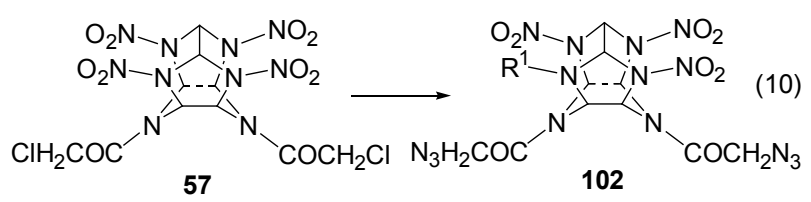

2006 年, 孟征等 ${ }^{[58]}$ 以 32 为底物, 与 $\alpha$-氯丙酰氯反 应, 制得了化合物 $\mathbf{1 0 3}$, 得率为 $88 \%$. 化合物 103 在硝硫 混酸中硝化得到含化合物 104 (实际未分离表征)的混合 物, 而直接进行叠氮化反应制得了化合物 105 (Scheme 12).

2008 年, Duddu 等 ${ }^{[59]}$ 以 98 为原料与 $\mathrm{ClCH}_{2} \mathrm{COCl}$ 在 加热条件下反应，制得了化合物 106,106 又与 $\mathrm{NaN}_{3}$ 在
丙酮中回流反应制得了化合物 107 (Scheme 13).
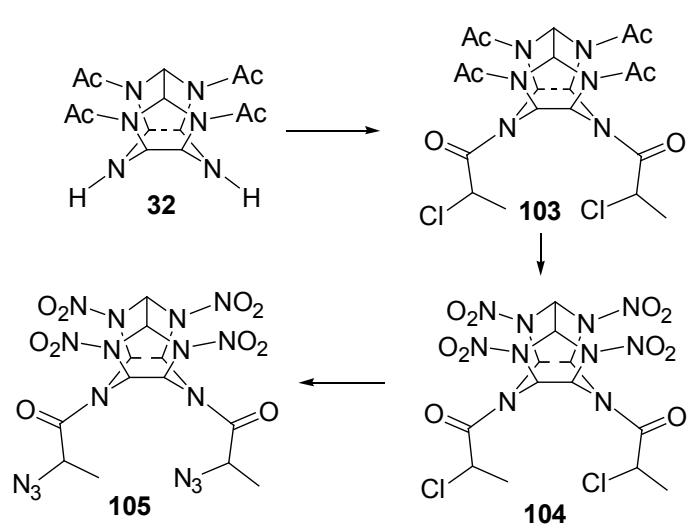

Scheme 12

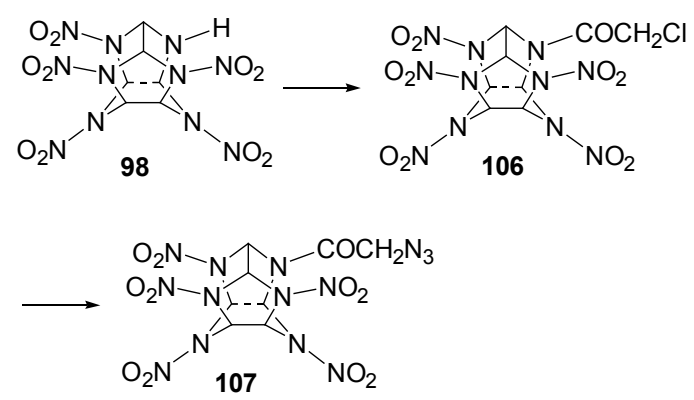

Scheme 13

\section{4 氧杂异伍兹烷}

除了在异伍兹烷笼体上引入氮原子外，也有研究者 将氧原子引入到笼体上，这不但能增加笼体的氧平衡， 还可以增加化合物密度.

早在 1979 年, 于永忠教授 ${ }^{[60}$ 就合成出了 4,10 -二硝 基-4,10-二氮杂-2,6,8,12-四氧杂四环十二烷(108), 简称 TEX. 并指出, 用硝胺基团替代该分子中氧原子所得的 化合物, 其综合性能可能超过 HMX, 将成为新一代高 能密度材料. 这一设想果然在 1987 年由美国 Neilsen 所 实现，即前文中的 CL-20.

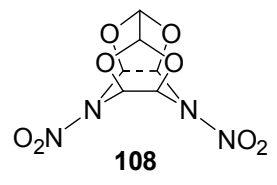

TEX 晶体密度为 $1.99 \mathrm{~g} / \mathrm{cm}^{3}$, 标准条件下撞击感度 为 $44 \%$, 摩擦感度为 $8 \%$; TEX 爆轰性能良好, 计算爆速 $8170 \mathrm{~m} \cdot \mathrm{s}^{-1}$, 爆压 $31.4 \mathrm{GPa}$, 是很有前途的钝感高能炸 药. 目前, 对 TEX 在熔铸炸药、推进剂等方面的应用进 行了大量研究，取得了一定的成果.

1990 年, Ramakrishnan 等 ${ }^{[61]}$ 由 1,4-二甲酰基-2,3,5,6四羟基哌嗪(THDFP)和三聚乙二醛硝化得到 TEX, 但该 
合成工艺所需反应时间较长, 且产物纯度低, 后处理工 艺复杂.

1993 年, Highsmith 等 ${ }^{[62]}$ 对 Ramakrishnan 的工艺进 行了优化, 降低了反应温度, 增加了产物纯度, 工艺步 骤也大量简化.

2003 年, Sanderson 等 ${ }^{[63]}$ 改进工艺条件, 在反应中仅 使用硝酸，且控制反应温度，得到纯度高于 $99.5 \%$ 的 TEX.

李战雄 ${ }^{[64]}$ 、徐容等 ${ }^{[65]}$ 也对 TEX 的合成工艺进行了 研究，在反应机理、产品得率以及纯度方面取得进展.

除了含有四个氧原子的 TEX 外, 也有含有一个或 两个氧原子异伍兹烷的报道.

2000 年, Jacob 等 ${ }^{[66]}$ 从 CL-20 粗品中分离得到两种 新的异伍兹烷, 2,4,8,10,12-五硝基-6-氧杂-2,4,8,10,12-六 氮杂异伍兹烷(109)和 4,6,8,10-四硝基-2,12-二氧杂4,6,8,10-四氮杂异伍兹烷(110).
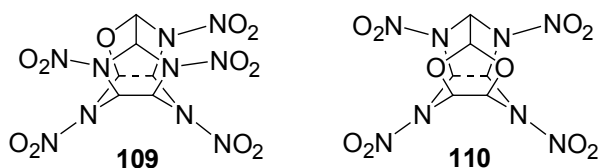

2005 年, Gatilov 等 ${ }^{[67]}$ 对 2,4,6,10-四硝基-2,4,6,10-四 氮杂 $-8,12$-二氧杂异伍兹烷 (111) 的两种晶型以及 $2,4,8,10$ - 四硝基-2,4,8,10-四氮杂- 6,12 -二氧杂异伍兹烷 (112)进行了详细的晶体结构表征, 其中 111 的晶体密度 达到 $2.014 \mathrm{~g} / \mathrm{cm}^{3}$, 是潜在的高能量密度化合物. 但文献 中并没有给出合成方法或来源.
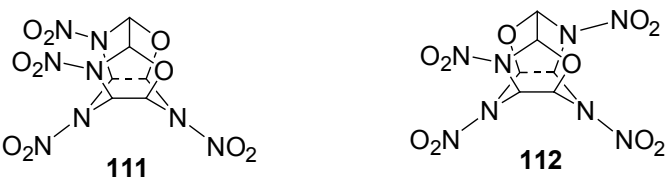

2006 年, Kerscher 等 ${ }^{[8]}$ 在制备化合物 17 时, 分离出 一个副产物 2,4,6,8,10-(五氟)苠基-2,4,6,8,10-六氮杂-12氧杂异伍兹烷(113), 并对其进行了表征.

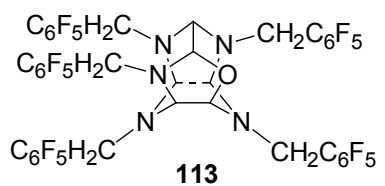

2006 年, 孙成辉等 ${ }^{[68]}$ 用 32 与甲醛反应, 得到了一 种新的异伍兹烷衍生物，3,5,9,11-四乙酰基-14-氧杂$1,3,5,7,9,11$-六氮杂十五烷(79). 2008 年，作者 ${ }^{[69]}$ 用乙醛、 氯乙醛取代甲醛得到化合物 13,15-二甲基-3,5,9,11-四乙 酰基-14-氧杂-1,3,5,7,9,12-六氮杂-十五烷(114)，13,15-
二氯甲基-3,5,9,11-四乙酰基-14-氧杂-1,3,5,7,9,11-六氮 杂五环 $\left[5.5 \cdot 3 \cdot 0^{2,6} .04,10.0^{8,12}\right]$ 十五烷(115) (Eq. 11). 这三 种化合物都有望作为新型含能化合物的合成前体.

$$
\begin{aligned}
& 114: \mathrm{R}=\mathrm{CH}_{3} \\
& 115: \mathrm{R}=\mathrm{CH}_{2} \mathrm{Cl}
\end{aligned}
$$

\section{5 结语与展望}

二十多年来，围绕着 CL-20 的合成以及寻找新的笼 形含能化合物，运用不同的反应类型已经合成了一百多 种异伍兹烷衍生物. 其中多种衍生物可以作为 CL-20 合 成的前体，将 CL-20 合成之初的昂贵价格降为可接受范 围; 同时也合成了一些有应用潜力的新的笼形含能化合 物.

异伍兹烷笼体结构很容易裂解，笼体上的六个取代 基反应活性也不尽相同，因此，异伍兹烷衍生物的合成 反应既要避免采用过强的反应条件造成笼体分解，又要 保证较高的反应能力使六个取代位置均发生转化, 在此 过程中发展了很多温和、高效的反应，促进了合成化学 的进步

但是，针对异伍兹烷衍生物的合成也存在以下问 题：(1)作为直接搭成笼子的胺醛缩合反应，适合的胺类 尚不够多，反应得率不高；(2)氧化反应的选择性和效率 不高; (3)在反应中有效保持异伍兹烷笼体不被破坏的方 法和手段不多.

因此，针对上述问题发展高效的催化体系、新的反 应试剂以及合成方法，是今后的研究重点. 相信随着研 究的进行，定能通过缩合反应直接合成出酰基取代的六 氮杂异伍兹烷硝化前体, CL-20 的合成必将会步骤减少、 成本降低，新的性能优异的异伍兹烷笼形含能化合物必 将会出现

\section{References}

[1] Qiu, L. M.; Gong, X. D.; Zheng, J.; Xiao, H. M. J. Hazard. Mater. 2009, 166, 931.

[2] Lauderdale, W. J.; Slwell, W. A. Air Force Astronautics Laboratory, Report AFAL CP-87-002, September, 1987, and subsequent reports.

[3] Nielsen, A. T.; Nissan, R. A.; Vanderah, D. J. J. Org. Chem. 1990 $55,1459$.

[4] Zhang, M. X.; Eaton, P. E.; Gilardi, R. Angew. Chem., Int. Ed. 2000, 39, 401.

[5] Simpson, R. L.; Urtiew, P. A.; Ornellas, D. L.; Moody, G. L.; Scribner, K. J.; Hoffmann, D. M. Propellants, Explos., Pyrotech. 1997, 22, 249. 
[6] Nielsen, A. T. US 5693794, 1997 [Chem. Abstr. 1997, 128, 36971].

[7] Klapötke, T. M.; Krumm, B.; Piotrowski, H.; Polborn, K.; Holl, G. Chem. Eur. J. 2003, 9, 687.

[8] Kerscher, T.; Klapötke, T. M.; Krumm, B.; Polborn, K.; Scherr, M. J. Fluorine Chem. 2006, 127, 1030.

[9] Gottlieb, H. E.; Cohen, S.; Gottlieb, L. Chem. Eur. J. 2010, 16, 5568.

[10] Cagnon, G.; Eck, G.; Herve, G.; Jacob, G. US 7279572, 2004 [Chem. Abstr. 2004, 142, 6566].

[11] Li, X. L.; Sun, C. H.; Zhao, X. Q.; Song, J. W. Chin. J. Energ. Mater. 2007, 15, 490 (in Chinese). (李新乐, 孙成辉, 赵信岐, 宋建伟, 含能材料, 2007, 15, 490.)

[12] Adamiak, J.; Maksimowski, P. Propellants, Explos., Pyrotech. 2009, 34, 315.

[13] Chapman, R. D.; Hollins, R. A. J. Energ. Mater. 2008, 26, 246.

[14] (a) Han, W. R.; Ou, Y. X.; Liu, J. Q.; Wang, G. L. Chin. Chem. Lett. 2004, 15, 1153.

(b) Han, W. R.; Ou, Y. X.; Liu, J. Q.; Chen, B. R. Chin. J. Org. Chem. 2005, 25, 1259 (in Chinese). (韩卫荣, 欧育湘, 刘进全, 陈博仁, 有机化学, 2005, 25, 1259.)

[15] Huang, X. M. S. Thesis, Beijing Institute of Technology, Beijing, 2005 (in Chinese). (黄兴, 硕士论文, 北京理工大学, 北京, 2005.)

[16] Han, W. R.; Ou, Y. X.; Zhang, X. H.; Huang, X.; Mou, W.; Gao, Y. L. Chin. J. Energ. Mater. 2008, 16, 153 (in Chinese). (韩卫荣, 欧育湘, 张雪红, 黄兴, 牟微, 高岩磊, 含能材料, 2008, 16, 153.)

[17] Wardle, R. B.; Hinshaw, J. C. US 7129348, 2006 [Chem. Abstr. 2006, 145, 438653].

[18] Lu, L. Y.; Ou, Y, X.; Wang, J. L. Fine Chem. 2004, 21, 321 (in Chinese). (吕连营，欧育湘，王建龙，精细化工, 2004, 21, 321.)

[19] Wang, C.; Ou, Y. X.; Wang, J. L. Chem. World 2000, 9, 462 (in Chinese). (王才, 欧育湘, 陈博仁, 化学世界, 2000, 9, 462)

[20] Yang, Z. Y. Ph.D. Dissertation, Beijing Institute of Technology, Beijing, 1995 (in Chinese). (杨宗云, 博士论文, 北京理工大学, 北京, 1995.)

[21] Kodama, T.; Minoura, H.; Miyake, N.; Yamamatus, S.; Katsumata, T. WO 98/16529, 1998 [Chem. Abstr. 1998, 129, 6228].

[22] Bellamy, A. J. Tetrahedron 1995, 51, 4711.

[23] Ou, Y, X.; Xu, J. Q.; Chen, J. T.; Chen, B. R.; Zheng, F. P.; Jia, H. P.; Wang, C. Chem. J. Chin. Univ. 1999, 20, 561 (in Chinese). (欧育湘, 徐永江, 陈江涛, 陈博仁, 郑福平, 贾会平, 王才, 高 等学校化学学报, 1999, 20, 561.)

[24] Wardle, R. B.; Edwards, W. W. WO 97/20785, 1997 [Chem. Abstr. 1997, 127, 110983].

[25] Qiu, W. G.; Chen, S. S.; Yu, Y. Z. Chin. J. Chem. 1999, 17, 554.

[26] Chen, S. S.; Qiu, W. G.; Yu, Y. Z. Acta Armamentarii 2000, 21, 116 (in Chinese). (陈树森, 邱文革, 于永忠, 兵工学报, 2000, 21, 116.)

[27] Chen, S. S.; Qiu, W. G.; Yu, Y. Z. Chin. J. Explos. Propellants 2000, 23, 11 (in Chinese). (陈树森, 邱文革, 于永忠, 火炸药学报, 2000, 23, 11.)

[28] Qiu, W. G.; Chen, S. S.; Yu, Y. Z. Chin. J. Synth. Chem. 2002, 10, 220.

[29] Qiu, W. G.; Chen, S. S.; Yu, Y. Z. Chin. J. Explos. Propellants 2001, 24, 62 (in Chinese). (邱文革, 陈树森, 于永忠, 火炸药学报, 2001, 24, 62.)

[30] Pang, S. P.; Yu, Y. Z.; Zhao, X. Q. Acta Armamentarii 2002, 23, 276 (in Chinese).

(庞思平, 于永忠, 赵信岐, 兵工学报, 2002, 23, 276.)
[31] Liu, J. Q.; Chen, S. S.; Yu, Y. Z.; Zhao, X. Q. Chin. J. Explosives Propellants 2003, 26, 5 (in Chinese). (刘江强, 陈树森, 于永忠, 赵信岐, 火炸药学报, 2003, 26, 5.)

[32] Pang, S. P.; Yu, Y. Z.; Zhao, X. Q. Propellants, Explos., Pyrotech. 2005, 30, 442.

[33] Li, Y. Y.; Wang, L.; Lin, Q. H.; Ma, Q. L.; Pang, S. P.; Yu, Y. Z. J. Beijing Institute Technol. 2010, 19, 476.

[34] Liu, J. Q.; Chen, S. S.; Yu, Y. Z.; Zhao, X. Q. Chin. J. Energ. Mater. 2002, 10, 145 (in Chinese).

(刘江强, 陈树森, 于永忠, 赵信岐, 含能材料, 2002, 10, 145.)

[35] (a) Liu, L. H.; Ou, Y. X.; Chen, B. R.; Xu, Y. J.; Lei, X. Y. Chin. J. Explos. Propellants 2000, 23, 50 (in Chinese).

(刘利华, 欧育湘，陈博仁，徐永江，雷向阳，火炸药学报，2000, 23, 50.)

(b) Liu, L. H.; Ou, Y. X.; Wang, J. Q.; Chen, B. R.; Lei, X. Y. Chin. J. Explos. Propellants 2001, 24, 16 (in Chinese).

(刘利华, 欧育湘，王建全，陈博仁，雷向阳，火炸药学报，2001, 24, 16.)

[36] Liu, J. Q.; Ou, Y. X. Acta Armamentarii, 2006, 27, 920(in Chinese). (刘进全，欧育湘，兵工学报, 2006, 27, 920.)

[37] Wu, X. G.; Wang, Y. F.; Ou, Y. X.; Liu, J. Q. Chin. J. Energ. Mater. 2006, 14, 343 (in Chinese).

(吴相国, 王艳飞, 欧育湘, 刘进全, 含能材料, 2006, 14, 343.)

[38] Lu, L. Y.; Ou, Y. X.; Wang, J. L.; Chen, B. R. Fine Chem. 2003, 20, 577(in Chinese).

(吕连营, 欧育湘, 王建龙, 陈博仁, 精细化工, 2003, 20, 577.)

[39] Kodama, K.; Masahiro, T.; Masanori, I. WO 96/23792, 1996 [Chem. Abstr. 1996, 125, 275920].

[40] Qian, H.; Ye, Z. W.; Lv, C. X. Lett. Org. Chem. 2007, 4, 482.

[41] Pang, S. P.; Yu, Y. Z.; Zhao, X. Q. Chin. J. Explos. Propellants 2002, 25, 27 (in Chinese). (庞思平, 于永忠, 赵信岐, 火炸药学报, 2002, 25, 27.)

[42] Pang, S. P.; Yu, Y. Z.; Zhao, X. Q. Chin. J. Energ. Mater. 2002, 10, 1 (in Chinese). (庞思平, 于永忠, 赵信岐, 含能材料, 2002, 10, 1.)

[43] Liu, J. Q.; Chen, S. S.; Yu, Y. Z.; Zhao, X. Q. Chin. J. Explos. Propellants 2003, 26, 60 (in Chinese). (刘江强, 陈树森, 于永忠, 赵信岐, 火炸药学报, 2003, 26, 60.)

[44] Hamilton, R. S.; Sanderson, A. J.; Wardle, R. B.; Warner, K. F. In Proceedings of 31st International Annual Conference of ICT, Karlsruhe, 2000, p. 21-1.

[45] Cannizzo, L.; Hamilton, S.; Sanderson, A.; Wardle, R.; White, S. In Proceedings of 32st International Annual Conference of ICT, 2000, p. 108-1.

[46] Bellamy, A. J.; MacCuish, A.; Golding, P.; Mahon, M. F. Propellants, Explos., Pyrotech. 2007, 32, 20.

[47] Fang, T.; Sun, C. H.; Zhao, X. Q. Chin. J. Energ. Mater. 2004, 12, 291 (in Chinese). (方涛, 孙成辉, 赵信岐, 含能材料, 2004, 12, 291.)

[48] Sun, C. H.; Fang, T.; Yang, Z. Y.; Bai, J. H.; Feng, Z. W.; Liu, J.; Li, L.; Ma, C. P.; Chen, Z. L.; Zhao, X. Q. Chin. J. Energ. Mater. 2009, 17, 161 (in Chinese).

(孙成辉, 方涛, 杨宗云, 白军红, 冯泽旺, 刘娟, 李莉, 马鹏常, 陈联忠, 赵信岐, 含能材料, 2009, 17, 161.)

[49] Chen, L. Q. M. S. Thesis, Beijing Institute of Technology, Beijing, 2008 (in Chinese). (陈留群, 硕士论文, 北京理工大学, 北京, 2008.)

[50] He, J. M. S. Thesis, Beijing Institute of Technology, Beijing, 2009 (in Chinese).

(贺晶, 硕士论文, 北京理工大学, 北京, 2009.)

[51] Shi, R. M. S. Thesis, Beijing Institute of Technology, Beijing, 2008 
(in Chinese).

(施瑞，硕士论文，北京理工大学，北京，2008.)

[52] Cai, S. L. M. S. Thesis, Beijing Institute of Technology, Beijing, 2008 (in Chinese).

(蔡水兰, 硕士论文, 北京理工大学, 北京, 2008.)

[53] Sun, C. H.; Zhao, X. Q.; Li, Y. C.; Pang, S. P. Chin. Chem. Lett. 2010, 21, 572.

[54] Bellamy, A. J.; MacCuish, A.; Golding, P. Propellants, Explos., Pyrotech. 2003, 28, 157.

[55] Lukyanov, O. A.; Shlykova, N. I. Russ. Chem. Bull. Int. Ed. 2004, 53,566 .

[56] Duddu, R.; Dave, P. R.; Damavarapu, R.; Surapaneni, R.; Gilardi, R. Synth. Commun. 2005, 35, 2709.

[57] Wu, Y. K.; Ou, Y. X.; Liu, J. Q.; Chen, B. R. Propellants, Explos., Pyrotech. 2004, 29, 155.

[58] Meng, Z.; Ou, Y. X.; Liu, J. Q.; Wang, Y. F. Chin. J. Explos. Propellants 2006, 29, 65 (in Chinese). (孟征，欧育湘，刘进全，王艳飞，火炸药学报，2006，29，65.)

[59] Duddu, R.; Dave, P. R.; Damavarapu, R.; Surapaneni, R.; Gilardi, R.; Parrish, R. Synth. Commun. 2008, 38, 767.

[60] 陈福波, 段宝如, 于永忠, 兵器工业部二一四所研究报告汇编, 1980, pp. 29 33.
[61] Ramekrishnan, V. T.; Vedachalam, M.; Boyer, J. H. Heterocycles 1990, $31(3), 478$

[62] Highsmith, T. K.; Edwards, W. W.; Wardle, R. B. US 5498711, 1995 [Chem. Abstr. 1995, 122, 290890].

[63] Sanderson, A. J.; Hajik, R. M.; Highsmith, T. K.; Johnston, H. E. US 6512113, 2003 [Chem. Abstr. 2003, 138, 124633].

[64] Li, Z. X.; Ou, Y. X.; Chen, B. R. Chin. J. Energ. Mater. 2001, 9, 104 (in Chinese) (李战雄, 欧育湘, 陈博仁, 含能材料, 2001, 9, 104.)

[65] Xu, R.; Zhou, X. Q.; Zeng, G. Y.; Liu, C. Chin. J. Explos. Propellants 2006, 29, 26(in Chinese). (徐容, 周小青, 曾贵玉, 刘春, 火炸药学报, 2006, 29, 26.)

[66] Jacob, G; Lacroix, G; Destombes, V. In Proceedings of 31st International Annual Conference of ICT, Karlsruhe, 2000, pp. 106-1 106-12.

[67] Gatilov, Y. V.; Rybalova, T. V.; Efimov, O. A.; Lobanova,; A. A.; Sakovich, G. V.; Sysolyatin, S. V. J. Struct. Chem. 2005, 46, 566.

[68] Sun, C. H.; Zhao, X. Q. J. Chem. Crystallogr. 2006, 36, 255.

[69] Sun, C. H.; Zhao, X. Q. Chin. J. Energ. Mater. 2008, 16, 669 (in Chinese).

(孙成辉，赵信岐，含能材料, 2008, 16, 669.)

(Sun, H.; Li, L.) 